\title{
ENERGY DISTRIBUTION OF TWO-ELECTRON IONIZATION OF HELIUM IN AN INTENSE LASER FIELD
}

\author{
R. LAFON ${ }^{1}$, J. L. CHALOUPKA ${ }^{1}$, B. SHEEHY ${ }^{1}$ AND L. F. DIMAURO ${ }^{1}$ \\ Brookhaven National Laboratory \\ Upton, NY 11973 USA \\ P.M. PAUL ${ }^{2}$, P. AGOSTINI ${ }^{2}$ \\ SPAM, Centre d'Etudes de Saclay \\ 91191 Gif Sur Yvette, France \\ AND \\ K. C. KULANDER ${ }^{3}$ \\ Lawrence Livermore National Laboratory \\ Livermore, CA 94551 USA
}

\section{Introduction}

It is well known that a neutral atom interacting with a strong laser field will ionize at sufficiently high intensity even for photon energies well below the ionization threshold. When the required number of photons becomes very large, this process is best described by the suppression of the Coulomb barrier by the laser's oscillating electric field, allowing the electron to tunnel into the continuum. As the laser intensity is increased, more tightly bound electrons may be successively liberated by this mechanism. Such a sequential multiple ionization, long accepted as a reasonable approach to the formidable problem of a multielectron atom interacting nonperturbatively with an intense electromagnetic field, provides fair estimates of the various charge state appearance intensities[1] while the tunneling rates are in excellent agreement with single ionization yields[2]. However, more accurate measurements revealed systematic and very large deviations from the tunneling rates $[2,3,4,5]$ : near appearance intensity under standard experimental conditions, the observed double ion yield is several orders of magnitude larger than predicted by the sequential ratc. It soon bccame clear that electrons could not be considered as independent and that electron-electron correlation had to be taken into account. Dynamic correlations have been 
considered in several theories. First qualitatively in the shakeoff model[4]; then empirically through the e-2e cross-section in the quantum/classical three-step model[6] (tunnel ionization, acceleration by the oscillating electric field and $\mathrm{e}-2 \mathrm{e}$ recollision with the ion); recently through the so-called intense field many-body-S-matrix theory[7] and a purely empirical model of collective tunnel ionization[8]. The validity of these ideas has been examined using numerical models $[9,10,11,12,13]$. The measurement of total ion yields over a dynamic range exceeding ten orders of magnitude[2], a major breakthrough made possible by the availability of high-repetition rate lasers at the beginning of the 90 's, was for a long time the only quantitative data to confront theory.

Recently, experiments have succeeded in collecting new information. Almost simultaneously, Cold-Target-Recoil-Ion-Momentum (COLTRIM) [14, 15] and electron-ion coincidence time-of-flight measurements $[16,17]$ were applied to the process of double-ionization in a strong field. The ion recoil momentum distribution, obtained in neon[14] and helium[15], have been interpreted as a signature of the interelectron correlation but also of the dressing of the two-electron final state[18]. Electron-ion momentum distributions taken in argon[17] have clearly revealed the tendency for the two electrons to be ejected on the same side of the nucleus with a similar momentum. Similarly, Witzel et al.[16] measured a "hot" electron energy distribution from xenon in coincidence with the doubly charged ion.

Obviously, our understanding of the strong-field double ionization of helium would benefit from a coincidence measurement. However, this measurement has been hindered by a unique set of experimental difficulties specific to helium. In this paper, we present time-of-flight energy spectra of electrons generated in the strong-field double ionization of helium and detected in coincidence with the corresponding charge state of the ion. The behavior of the two-electron distributions are examined as a function of intensity for $0.78 \mu \mathrm{m}, 100$ fs pulses. Helium is the ideal candidate for the study of non-sequential (NS) double ionization, its simple structure affords theoretically tractable and greatly reduces the ambiguities present in more complex atoms due to higher-order "resonant" processes. Furthermorc, strong-ficld ionization of helium has been the subject of intensive experimental and theoretical investigations, resulting in the most comprehensive understanding in all of intense laser-atom interaction. Previous studies[2, 19, 20] have shown quantitative agreement with quasi-classical models which validated the strong field limit of single electron rescattering dynamics. However, the two electron dynamics have remained unclear. In our experiment, the electrons detected in coincidence with a doubly ionized helium atom show a remarkable enhancement at high energies compared to the electrons involved in the single ionization process. We will show that, 


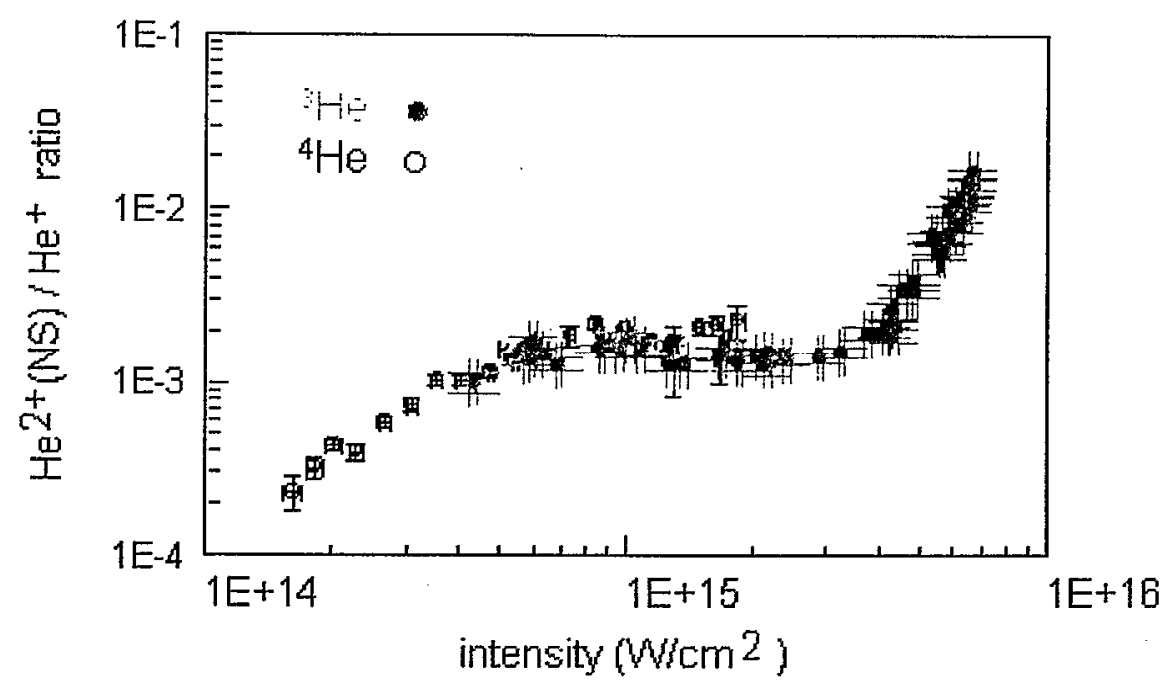

Figure 1. Plot of the ratio of $\mathrm{He}^{2+} / \mathrm{He}^{+}$for ${ }^{4} \mathrm{He}$ (squares) and ${ }^{3} \mathrm{He}$ (circles) as a function of intensity for $0.78 \mu \mathrm{m}$ excitation. For helium, the ratio in the NS region is never larger than 0.002 .

classically, this can only be due to rescattering in the backward direction.

\section{Experimental Issues}

A measure of the two-electron energy distribution for helium has been elusive since it poses unique experimental challenges. The most obvious is illustrated in Fig. 1. A plot of the intensity dependence of the $\mathrm{He}^{2+} / \mathrm{He}^{+}$ ratio not only provides a sensitive measure of the NS dynamics but a immediate look at the problem of recording the two-electron distribution. The ratio of double-to-single ionization events is never larger than 0.002 or 1:500. Although this is also a problem for other atoms, the NS ratio in helium is at least an order of magnitude smaller. Thus, even in an ideal experimental system, e.g. perfect particle detectors and zero contamination, the problem remains the detection of the two electrons of interest from the plethora of single ionization.

In order to generate an electron distribution that correlates to a specific ion species and charge state, an ion charge-to-mass spectrum must be generated along with an electron time-of-flight spectrum. By detecting only one ion and one or more electrons in coincidence, it can be determined with some level of certainty that the detected ion and electrons were involved in the same ionization process. This level of certainty manifests itself in the 
number of "true" counts that are accumulated (where the electrons and ion are positively correlated) versus the number of "false", or accidental counts (where the electrons and ions are not correlated)[21]. This true-tofalse ratio can be primarily improved in two ways. First, the collection and detection efficiencies of the ions and electrons must be made as close to unity as possible. In fact, if the ion efficiency is made to be perfect, then there will never be an opportunity for accidental coincidence counts. Second, the overall count rate can be lowered, making the likelihood of two ionization events per single laser shot as small as reasonable. For a certain set of experimental conditions, the true-to-false ratio can be calculated and used in the interpretation of the data.

In doing the coincidence experiment with helium, the general requirement to keep the overall count rate low is complicated by two factors. First, the ion yield of the second charge state with respect to the first is extremely low in the non-sequential region $(<1 / 500)$. Therefore, if the detection rate of $\mathrm{He}^{+}$is kept relatively low (e.g., at an average of one detection per five laser shots), then the detection rate of $\mathrm{He}^{2+}$ will drop below one hit per 2500 shots. Depending on the electron detection efficiency, the rate of coincidence counts will be much lower still. In order to accumulate a significant amount of data, it is important to use a laser system at a high repetition rate and in a stable fashion over the course of many hours or even days. Second, the ionization threshold for the first charge state of helium is rather high ( $24 \mathrm{eV}$, corresponding to a saturation intensity for tunnel ionization of $8 \times 10^{14} \mathrm{~W} / \mathrm{cm}^{2}$ at $0.78 \mu \mathrm{m}$ and $100 \mathrm{fs}$ ). For many laser systems, this does not present a problem in terms of generating the peak intensity. Rather, it has the effect of enhancing the contribution of unwanted signal from the contaminants in the vacuum chamber. Since all the likely contaminants are ionized at much lower intensities, their contribution at the helium saturation intensity will come from a much larger focal volume. This effectively worsens the quality of the vacuum (e.g., the contribution from water is magnified by a factor of 250 at the helium saturation intensity). Clearly, the vacuum base pressure must be very low, and the purity of the helium introduced into the chamber must be as high as possible.

The laser used in this study was a titanium:sapphire chirped-pulse amplification system and has been described elsewhere[2]. It was operated at $1-2 \mathrm{kHz}$ repetition rate and produced $100-\mathrm{fs}$ pulses at a wavelength of 0.78 $\mu m$. The light was tightly focused to generate peak intensities as high as $10^{16} \mathrm{~W} / \mathrm{cm}^{2}$. The focal region was aligned to the center of a two-sided, pulsed-plate, ion-electron spectrometer (see Fig. 2). Electrons liberated in the ionization process are allowed to drift in a field-free region down the gold-plated flight tube towards the electron micro-channel plate (MCP) assembly. After a delay of $200 \mathrm{~ns}$, plates 5 and 7 are pulsed from ground to 


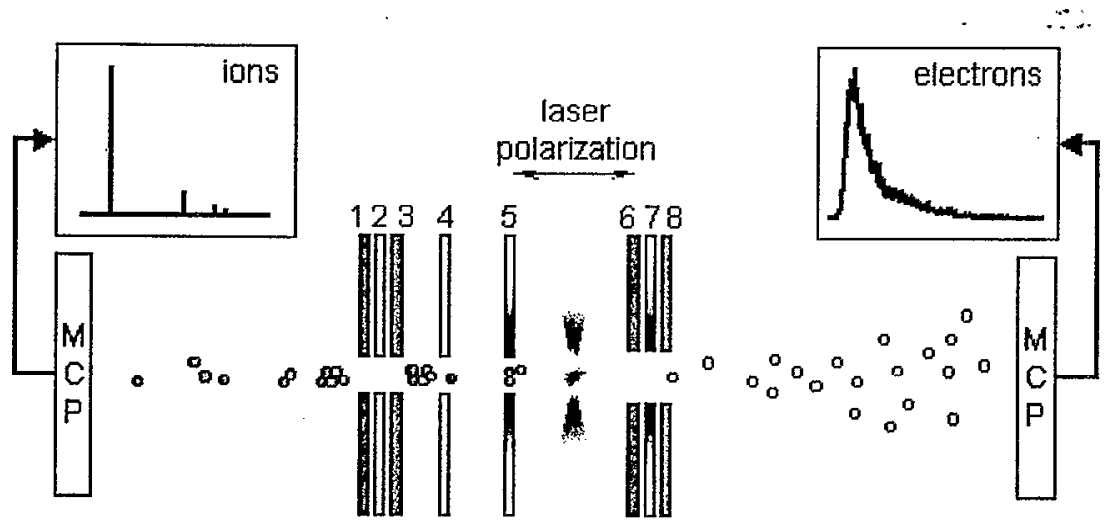

Figure 2. The two-sided electron-ion spectrometer. Electrons are allowed to drift in a static-field-free region towards the electron detector. Ions are extracted by pulsing the extraction plate to a negative voltage. Simultaneous detection of electrons and ions are performed for each laser shot.

$-75 \mathrm{~V}$ and $-150 \mathrm{~V}$, respectively. Plates 6 and 8 remain grounded. The voltage on plate 5 extracts the ions from the focal region, while the voltage on plate 7 blocks any secondary electrons generated by ion-plate impact from entering the electron flight tube. The delay of $200 \mathrm{~ns}$ is long enough to allow nearly all of the liberated electrons to drift into the protected, field-free flight tube before the turn-on of the plate voltages. The delay is also short enough that the ion displacement due to thermal motion does not affect their extraction towards the ion MCP. Both of these issues were verified in grounded and static-field tests. The electron detector subtends a solid angle of $10^{\circ}$ and has an absolute collection and detection efficiency of roughly $1 \%$. The voltages on plates 1 through 5 are tuned to optimize the extraction efficiency and resolution of the ion detector, giving it an absolute collection and detection efficiency of roughly $30 \%$. The spectrometer sat within an ultra-high vacuum chamber with a nominal base pressure of $10^{-10}$ torr. The gas to be studied was delivered via a leak valve into the cntire chamber, while continually pumping with turbo-molecular pumps. In our experiment, ${ }^{3} \mathrm{He}$ is used instead of ${ }^{4} \mathrm{He}$ to enhance the measurement sensitivity by removing the nearly degenerate $m / q$ ratio between ${ }^{4} \mathrm{He}^{2+}$ and $\mathrm{H}_{2}^{+}$, a major contaminant. To aid in the reduction of water contamination through the gas line, a liquid-nitrogen-cooled coil was used at times.

\section{Coincidence Measurement}

The spectrometer was first tested in non-coincidence experiments. Previously studied electron spectra and ion ratio curves were reproduced in both static field and pulsed plate modes. Then, to test the apparatus in 

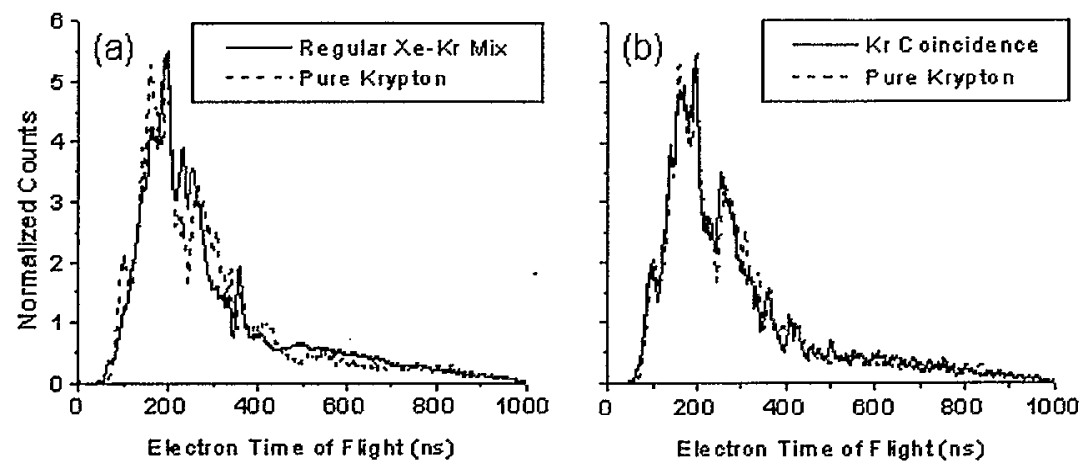

Figure 3. The $\mathrm{Xe} / \mathrm{Kr}$ gas mix test. a) The ordinary electron spectrum generated with the gas mix (solid line) and the pure krypton spectrum (dashed line). b) The krypton coincidence spectrum taken with the gas mix (solid line) and the pure krypton spectrum (dashed line). All curves are normalized by their intcgratcd valucs, smoothed from 1-nsec to 10 -nsec resolution and uncorrected.

a coincidence-style experiment, a controlled gas mix of krypton and xenon was used. First, the ordinary electron energy spectra of pure krypton and pure xenon were taken at an intensity of $4 \times 10^{13} \mathrm{~W} / \mathrm{cm}^{2}$. Next, a coincident measurement using the gas mix was performed, giving a xenon:krypton ion count rate of 8:1 and an overall xenon detection rate of one hit per ten laser shots. The data from 65.9 million laser shots was accumulated at a repetition rate of $1 \mathrm{kHz}$ over a total run time of nearly 20 hours. It consists of the total, non-coincident electron and ion spectra, as well as the ion and electron arrival times when only one ion is detected. With this information, it is straightforward to compile the coincident electron spectrum for any of the detected ions. Figure 3(a) shows the total electron time-of-flight spectrum (solid line) generated with the gas mix, as well as the krypton spectrum (dashed line) generated with the pure gas. Both curves are normalized by their integrated values and are smoothed from 1-nsec to 10-nsec resolution. The curves are dissimilar since only $1 / 10$ of the total spectrum is made up of electrons from krypton, their features are hidden by the more abundant xenon electrons. Figure 3(b) shows the krypton coincidence spectrum (solid line), which has been extracted from the total spectrum, and the pure krypton spectrum (dashed line). The much improved agreement is unmistakable. The calculated true:false ratio for this test was roughly $3: 1$, and the curve shown is uncorrected and is made up of a total of 7375 coincident electron hits. This test shows, in a clear and controlled fashion, the effectiveness of our coincidence technique.

Coincidence data using helium was taken at two intensities: the single 
ionization saturation intensity $\left(\mathrm{I}_{s a t}=8 \times 10^{14} \mathrm{~W} / \mathrm{cm}^{2}\right)$ and half of this value. Figure 4 shows the energy spectra for electrons detected in coincidence with the single and double ionization of ${ }^{3} \mathrm{He}$ at these two intensities. The curves are normalized by their integrated value, and the horizontal axis shows the electron energy as well as the ponderomotive potential $\left(\mathrm{U}_{p}\right)$ associated with the peak laser intensity. All of the curves are uncorrected for false counts and include representative error bars that reflect counting statistics and the true:false ratio. Figure 4(a) shows the energy spectra for electrons correlated to single (solid line) and double ionization (circles) at $I_{s a t}$. A total of 205 million laser shots were taken. The detection rate of ${ }^{3} \mathrm{He}^{+}$was roughly one in five, and the rate of background ions was one in three. The ratio of double to single ionization was 0.0013 . These high count rates were used to improve the counting statistics on the double ionization electron spectrum, resulting in an overall true:false ratio that was calculated to be roughly 1:1. However, for the high energy electrons, where the abundance of "false" electrons is very small, the true:false ratio is much greater. For example, at $100 \mathrm{eV}$, the effective true:false ratio is 100:1. This is simply due to the paucity of high-energy electrons that can contribute to accidental counts. As a result, correcting the double ionization spectrum by subtracting away false counts has very little effect on the electron distribution beyond $50 \mathrm{eV}$. Figure 4(b) shows the energy spectra generated at $1 / 2 \mathrm{I}_{\text {sat }}$. A total of 190 million laser shots were taken. The detection rate of ${ }^{3} \mathrm{He}^{+}$was roughly one in four, and the rate of background ions was one in five. The ratio of double to single ionization was 0.00059. Again, the overall true:false ratio was calculated to be roughly $1: 1$, but, as before, the effective ratio at high energies is much improved. The dramatic enhancement of these spectra at high energies for electrons involved in the double ionization process is readily apparent at both intensities. In fact, when scaled to $U_{p}$, it appears that the NS distributions are nearly identical.

\section{Discussion}

The double ionization electron spectra in Fig. 4 show some remarkable differences compared to the single ionization spectra. The single ionization features are well understood. The rapidly decreasing distribution for energies smaller than $2 U_{p}$ is a result of the tunneling rate and the drift velocity corresponding to the phase at which the electron is set free in the field[6]. The long plateau extending to $10 U_{p}$ is due to the electrons that are born after the peak of the field that return to the core where they backscatter elastically and are subsequently accelerated by the field to high energies. In contrast, the double ionization spectra of Fig. 4 show a much slower rate of decrease. They extend at least to $4 U_{p}$ with profiles similar to that 


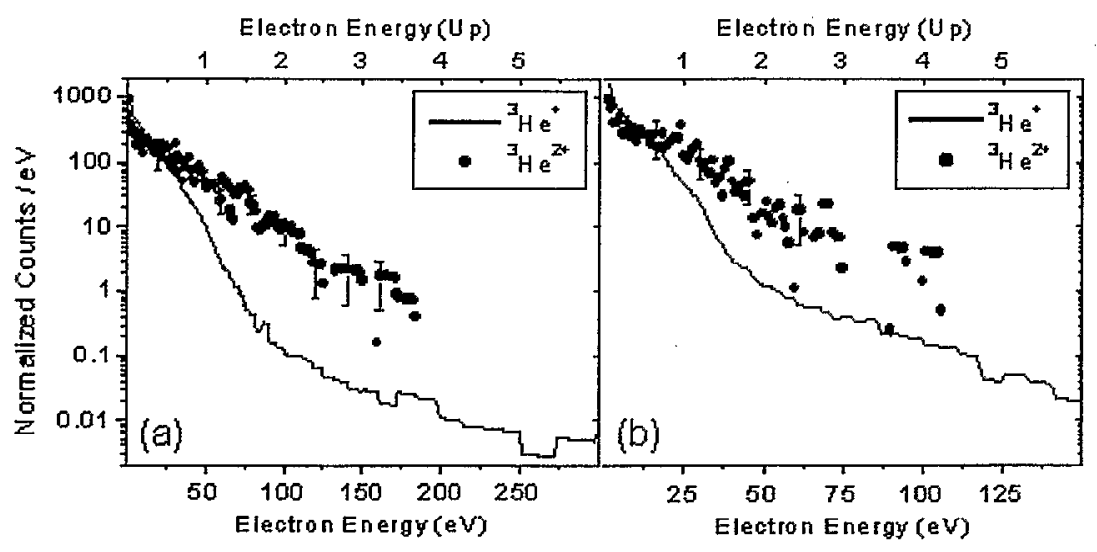

Figure 4. The energy spectra for electrons detected in coincidence with ${ }^{3} \mathrm{He}^{+}$(dashed line) and ${ }^{3} \mathrm{He}^{2+}$ (solid line) at a) the single ionization saturation intensity and b) one-half of the saturation intensity. The enhancement of the high-energy electrons coming from the double ionization process is dramatic at both intensities, and appears to scale with the ponderomotive potential $\left(U_{p}\right)$ associated with the peak intensity. Representative error bars reflect the counting statistics and the true:false ratio. All curves are uncorrected for false counts. Correcting the double ionization curves has a negligible effect on electrons above one $\mathrm{U}_{p}$.

of the rescattering plateau of single ionization. The fact that the coincidence electrons can have energies beyond $2 U_{p}$ clearly implies the release of electrons into the field with an initial non-zero backward velocity. In the rescattering model[6], an electron returning to the core with a kinetic energy larger than the binding cncrgy of the second electron can liberate the second electron, giving rise to two electrons sharing the excess energy. The final electron energies depend on the amplitude and direction of the electron initial velocities, with the largest kinetic energies being reached for backward initial velocities. Measurements on e-2e triply differential cross sections (TDCS) for hydrogen and helium show that a substantial fraction of the electrons are scattered into the backward direction, particularly near threshold[22, 23]. Even at high collision energies, it is most likely that when one electron carries off most of the energy in the forward direction that the slower electron will emerge in the backward direction.

To illustrate the consequence of backscattering on the final electron energies, a simple 1D classical calculation was performed. Electron trajectories were initiated at all phases of the field, and were followed after the $c-2 c$ ionizing collision. Collision onergies larger than the separation energy $(40 \mathrm{eV})$ between the ground and first excited state in singly ionize helium are assumed to satisfy the condition for ionizing the second electron via 


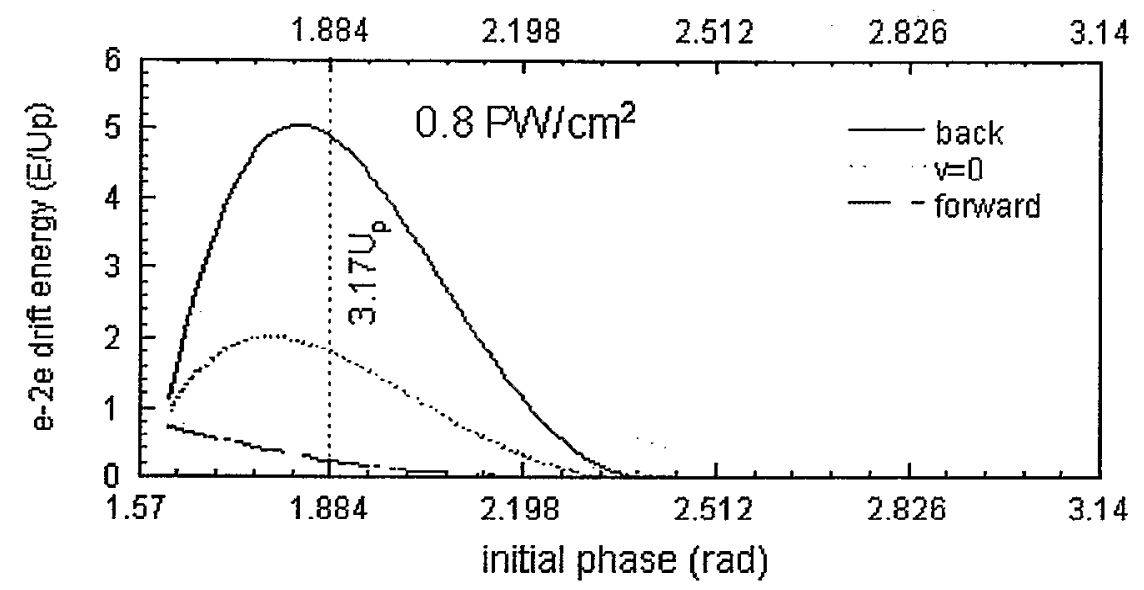

Figure 5. Plot of initial phase versus final drift energy after an inelastic e-2e collision. The shaded area indicates the initial phases having return energies equal to or greater than the $\mathrm{He}^{+}$binding energy of $40 \mathrm{eV}$. The results are calculated for an intensity of $0.8 \mathrm{PW} / \mathrm{cm}^{2}$. The dotted vertical line indicates the initial phase which produces the maximum return energy of $3.17 \mathrm{U}_{p}$.

an e-2e process. Those initial phases for an intensity of $0.8 \mathrm{PW} / \mathrm{cm}^{2}$ are shown by the shaded region in Fig. 5 . The excess energy is assumed to be taken by one electron either in the forward (sign of velocity unchanged by the collision) or backward (sign of velocity changed by the collision) direction and the final drift energy is calculated. The forward (dashed line) and back (solid line) scattering curves are plotted in Fig. 5, along with a curve (dotted line) assuming zero velocity after the e-2e process. The zero velocity curve has an expected upper bound of $2 \mathrm{U}_{p}$ energy. However, any initial velocity along the forward direction will result in a final drift energy less than $2 \mathrm{U}_{p}$. In fact at the saturation intensity, the final energy is substantially reduced with a maximum around $0.75 \mathrm{U}_{p}$. Clearly, the measured double ionization electron distributions in Fig. 4 have energies well above $2 U_{p}$ and the result in Fig. 5 shows that back scattering will produce energies up to $5 \mathrm{U}_{p}$. The calculation over-simplifies the problem but it unequivocally shows the essential role that backscattering must play in any rescattering e-2e process.

We conclude from these calculations, that the observed relatively flat electron energy distributions that extend out to $4 U_{p}$ reflect the high probability of the scattered or ionized electron emerging initially in the backward direction. Since this is known to be the case in field-free e-2e measurements, the dramatic differences between the single and double ionization electron spectra are not surprising. Our results, in agreement with recent measure- 
ments $[14,15,16,17]$ are therefore compatible only with a rescattering mechanism of NSDI. However, our measurements clearly show electron energies larger than $2 U_{p}$, implying drift motions with backward non-zero initial velocities. This is very different from that reported in argon[17].

In conclusion, our electron-ion coincidence measurements in helium, presented here for the first time, provide new support for the rescattering model of NSDI. They also provide new information about the maximum energy of the photoelectrons produced in the strong field NSDI of helium. This serves to emphasize the role of backward electron emission during the e-2e event in agreement with known TDCS in hydrogen and helium. It is expected that this work will stimulate full quantum calculations of the two-elèctron energy distribution.

\section{Acknowledgments}

The experiments were carried out at Brookhaven National Laboratory under contract No. DE-AC02-98CH10886 with the U.S. Department of Energy and supported by its Division of Chemical Sciences, Office of Basic Energy Sciences. P. A. acknowledge travel support from NATO under Contract No. SA.5-2-05(RG910678). This work was also carried out in part under the auspices of the U. S. Department of Energy at the Lawrence Livermore National Laboratory under contract No. W-7405-ENG-48.

\section{References}

1. Gibson, G., Luk, T.S. and Rhodes, C.K. (1990) Tunneling ionization in the multiphoton regime, Phys. Rev. A 41, pp. 5049-5052.

2. Walker, B. et al. (1994) Precision measurement of strong field double ionization of helium, Phys. Rev. Lett. 73, pp. 1227-1230.

3. L'Huillier, A., Lompre, L.A., Mainfray, G. and Manus, C. (1983) Multiply charged ions induced by multiphoton ansorption in rare gases at $0.53 \mu \mathrm{m}$, Phys. Rev. A 27, pp. 2503-2512.

4. Fittinghoff, D.N., Boltun, P. R., Chang, B. and Kulauder, K. C. (1992) Observation of nonsequential double ionization of helium with optical tunneling, Phys. Rev. Lett. 69, pp. 2642-2645.

5. Larochelle, S., Talebpour, A. and Chin, S.L. (1998) Non-sequential multiple ionization of rare gas atoms in a Ti:Sapphire laser field, $J$. Phys. B 31, pp. 1201-1214.

6. Corkum, P.B. (1993) Plasma perspective on strong field multiphoton ionization, Phys. Rev. Lett. 71, pp. 1994-1997.

7. Becker, A. and Faisal, F.H.M. (1999) Interplay of electron correlation and intense field dynamics in the double ionization of helium, Phys. Rev. A 59, pp. R1742R1745.

8.' Eichmann, U. et al. (2000) Collective multielectron tunneling ionization in strong fields, Phys. Rev. Lett. 84, pp. 3550-3553.

9. Watson, J.B. et al. (1997) Nonsequential double ionization of helium, Phys. Rev. Lett. 78, pp. 1884-1887.

10. Parker, J.S., Smyth, E.S. and Taylor, K.T. (1998) Intense-field multiphoton ionization of helium, J. Phys. B 31, pp. L571-L578. 
11. Kornberg, M.A. and Lambropoulos, P. (1999) Plotoelectron energy spectrum in "direct". two-photon double ionization of helium, J. Phys. B 32, pp. L603-L613.

12. Panfili, R., Szymanowski, C., Liu, W.-C. and Eberly, J.H. (2000) Spectroscopy in the neighborhood of the double ionization knee, in L.F. DiMauro, R.R. Freeman, K.C. Kulander (eds.), Multiphoton Processes, American Institute of Physics, New York, pp. 265-272.

13. Yudin, G. and Ivanov, M. (2000) Physics of correlated double ionization of atoms in intense laser fields: quasistatic tunneling limit, Phys. Rev. A, accepted.

14. Moshammer, R. et al. (2000) Momentum distributions of $\mathrm{Ne}^{\text {nt }}$ ions created by an intense ultrashort laser pulse, Phys. Rev. Lett. 84, pp. 447-450.

15. Weber, Th. et al. (2000) Recoil-ion momentum distributions for single and double ionization of helium in strong laser fields, Phys. Rev. Lett. 84, pp. 443-446.

16. Witzel, B., Papadogiannis, N.A. and Charalambidis, D. (2000) Charge-state resolved above threshold ionization, Phys. Rev. Lett. 85, pp. 2268-2271.

17. Weber, Th., et al. (2000) Correlated electron emission in multiphoton double ionization, Nature 405, pp. 658-661.

18. Becker, A. and Faisal, F.H.M. (2000) Interpretation of momentum distribution of recoil ions from laser induced nonsequential double ionization, Phys. Rev. Lett. 84, pp. 3546-3549.

19. Walker, B., Sheehy, B., Kulander, K.C. and DiMauro, L.F. (1996) Elastic rescattering in the strong field tunneling limit, Phys. Rev. Lett. 77, pp. 5031-5034.

20. Sheehy, B. et al. (1998) Single- and multiple-electron dynamics in the strong-field tunneling limit, Phys. Rev. A 58, pp. 39423952.

21. Stert, V., Radloff, W., Schulz, C.P. and Hertel, I.V. (1999) Ultrafast photoelectron spectroscopy: femtosecond pump-probe coincidence detection of ammonia cluster ions and electrons, Eur. Phys. J. D 5, pp. 97-106.

22. Roder, J., Ehrhardt, H., Bray, I. and Fursa, D.V. (1997) Absolute double differential cross sections for electron-impact ionization of helium, J. Phys. B 30, pp. 1309-1322.

23. Rioual, S. et al. (1998) Absolute (e,2e) cross sections for the electron-impact ionization of helium in energy sharing kinematics at $44.6 \mathrm{eV}, J$. Phys. B 31, pp. 3117-3127. 